\title{
Distichiasis: management with cryotherapy to the posterior lamella
}

\author{
B A O’Donnell, J Richard O Collin
}

\begin{abstract}
Distichiasis is the congenital condition of partial or complete accessory rows of eyelashes which exit from the posterior lid margin at or near the meibomian gland orifices. A series of 24 patients with distichiasis is presented with clinical features and results of treatment. The treatment modes of epilation, lid margin cryotherapy, or eyelid splitting cryotherapy to the posterior lamella are evaluated. The latter was found to effectively relieve symptoms without retreatment in $87 \%$ patients. Keratinisation of the lid margin was found at long term follow up in a few cases and retinoic acid has been used successfully in the treatment of this. Two previously unreported associations of distichiasis are Pierre Robin syndrome and idiopathic eyelid oedema. A family history of distichiasis was found in $\mathbf{5 0 \%}$ patients, and of distichiasis with lymphoedema in $30 \%$.
\end{abstract}

(Brf Ophthalmol 1993; 77: 289-292)

Method

All patients with distichiasis who have attended

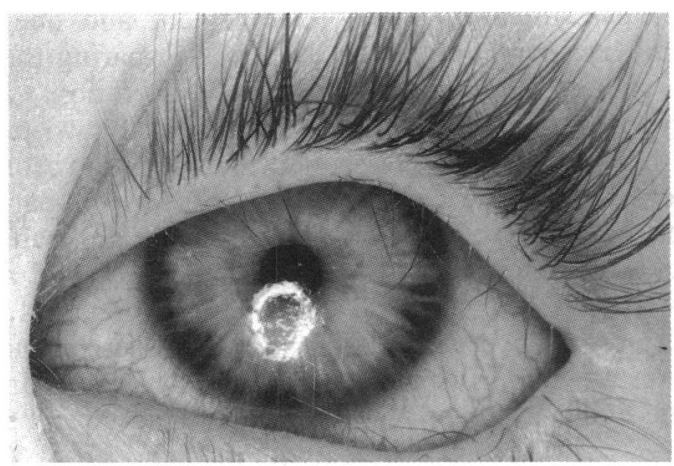

Figure 1 Eyelid with distichiasis: double row of lashes in left upper and lower lids.

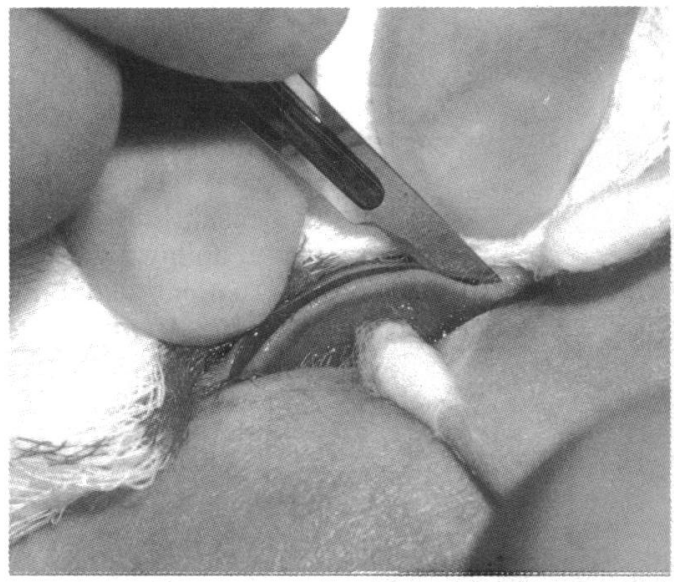

Figure 2 The lid is fixated while performing lid split at the grey line.
Moorfields Eye Hospital and the private practice of one of the authors over the past 13 years were retrospectively reviewed. Patient names were taken from a log kept of all patients with this diagnosis. Data were then gathered from hospital and consultant files. Patient referral was from ophthalmologists and general practitioners. The clinical features, presenting symptoms, family history, treatment, and clinical course were reviewed. Patients not recently reviewed were recalled for long term follow up.

Treatment, if required was in the form of epilation, electrolysis, lid margin cryotherapy, or lid margin splitting with cryotherapy to the posterior lamella. The decision to treat was made if symptoms were present, or if significant corneal staining or ulceration was observed. The type of treatment was chosen according to the extent of lid margin involved and history of previous failed treatment. Thus if relatively few distichiatic lashes were present, electrolysis only was performed. If less than a quarter lid margin extent was involved or previous electrolysis had failed, cryotherapy was applied. Cryotherapy was avoided in dark skinned patients and those

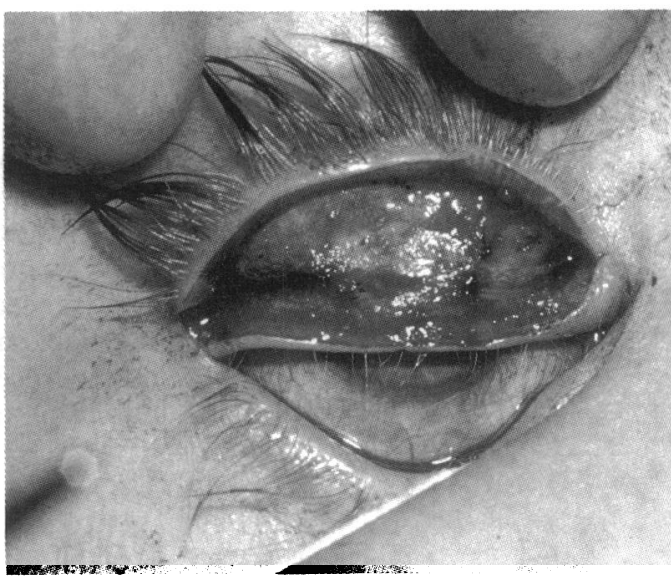

Figure 3 The anterior and posterior lamellae are separated.

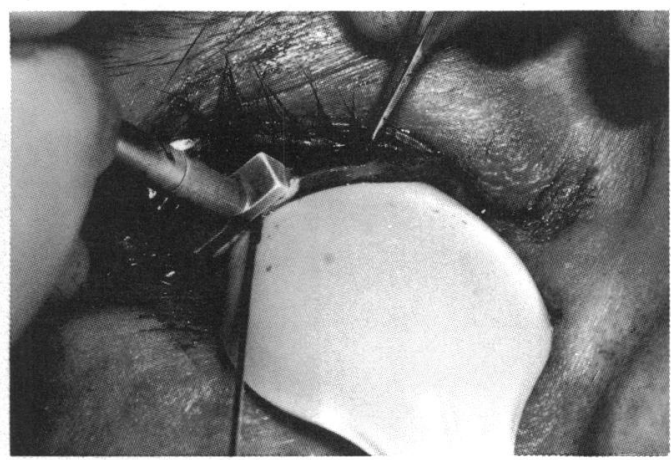

Figure 4 The cryoprobe applied to the posterior lamella. A thermocouple is placed between the lid and a plastic spoon which protects the cornea. 
Figure 5 The posterior lamella is advanced in relation to the anterior lamella at completion.

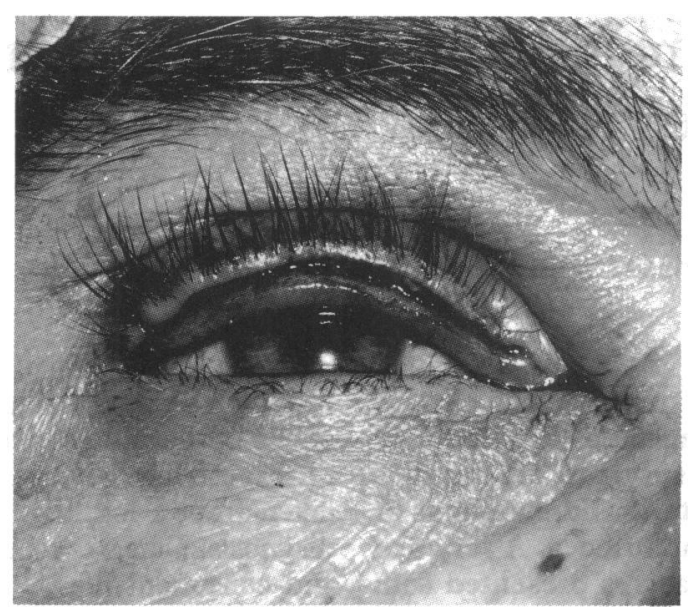

with dark lashes to avoid uncosmetic postoperative depigmentation. If more than half of the lid margin was involved (Fig 1), then lid splitting with posterior lamellar cryotherapy was performed. Treatment success was recorded if there was complete or near complete resolution of symptoms, complete or near complete removal of the distichiasis lashes, or disappearance of pretreatment fluorescein staining.

Surgery was performed with general anaesthesia in children and with either general or local anaesthesia in adults depending on patient preference and general health. Local anaesthesia was performed with a standard mixture of equal parts of $2 \%$ lignocaine with adrenaline and $0.5 \%$ marcaine.

\section{Surgical technique}

The surgical technique used is similar to that previously described by Anderson and Harvey ${ }^{1}$ in which the lid margin is split at the grey line (Figs 2,3 ) to enable a cryoprobe to freeze the posterior lamella without freezing the anterior lamella (Fig 4). However, in this series an anterior lamella recession is performed by releasing the lid retractors to enable the posterior lamella to be advanced $4 \mathrm{~mm}$ (Fig 5). ${ }^{2}$ This results in more reliable anterior lamellar recession than does simple recession of the skin and orbicularis muscle onto the anterior surface of

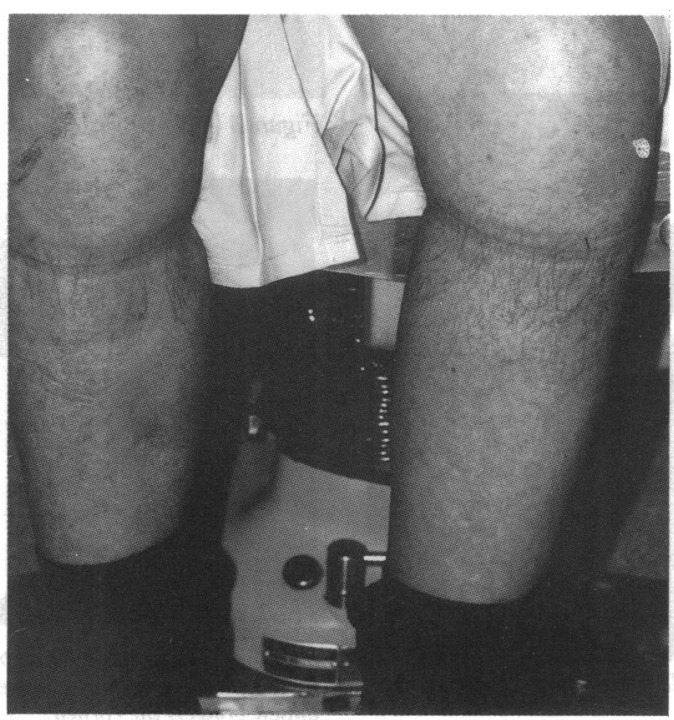

the tarsal plate. A double freeze-thaw technique is applied to the posterior lamella. The temperature is monitored with a thermocouple placed under the tarsus with a lamellar division to avoid the risk of necrosis of the tarsus. Freezing is applied for the time taken for the thermocouple to register $-20^{\circ} \mathrm{C}$. This rapid freezing is followed by slow thawing before re-freezing. ${ }^{3}$ The Collin cryoprobe (Keeler Ophthalmic Instruments), which has been specifically designed for eyelid margin cryotherapy, is used as it allows a greater length of lid margin to be evenly frozen than with an ordinary probe. Following lid split and posterior lamellar advancement, the anterior and posterior lamellae are sutured together with a running $6 / 0$ vicryl suture. Follow up treatment was at 1 week, 4 weeks, and 2 months, but was frequently varied on an individual basis according to patient travelling distance and postoperative comfort.

\section{Results}

Twenty four patients have attended Moorfields Eye Hospital with distichiasis over the past 13 year period. Nine patients were referred from other ophthalmologists and 13 patients were referred from general practitioners. Two patients attended as a result of family history or distichiasis. The average age of patients was $9 \cdot 7$ years with a range of 1.8 to 50 years. There were 11 male and 13 female patients. All except one patient were symptomatic. Symptoms reported were photophobia, lid swelling, conjunctival injection, lacrimation, discharge, and irritation.

All patients had four lid involvement with two exceptions: one with only right upper lid involvement and one with bilateral lower lid distichiasis sparing them upper lids. Recurrent corneal ulceration was present on initial examination in two patients with scarring and neovascularisation in one of these. Family history was recorded in all patients and $50 \%$ had at least one previous generation affected. Two sisters with distichiasis had a family history affecting four generations, and one patient had a family history with three generations affected. In both these families, autosomal dominant inheritance was evident. Two patients had co-existent lymphoedema (Fig 6): in one of whom the lymphoedema followed pregnancy. Two patients, mother and daughter, gave a history since early teenage, of eyelid swelling. Blepharoplasty was performed successfully in the mother. Other associated findings were Pierre Robin syndrome (one patient), telecanthus and epicanthus (one patient), and ptosis (two patients). The latter required bilateral brow suspension and anterior levator resection respectively, following treatment of the distichiasis. One patient had postoperative eyelid oedema following two anterior approach ptosis corrections, which was not present preoperatively.

Eight patients were treated initially at Moorfields with only electrolysis and/or cryotherapy. Two required subsequent lid split and cryotherapy. The remaining patients had complete removal of lashes although multiple treatments were required sometimes. One 
patient refused treatment, and two cases were treated elsewhere with lid splitting and cryotherapy and are not included in these results. Fifteen patients underwent lid splitting with posterior lamellar cryotherapy: three patients with all four lids, four with bilateral lower lids, and eight with bilateral upper lids. Five of these had had previous electrolysis and/or cryotherapy (including patients treated initially elsewhere). Of the group with lid splitting, all patients had significant improvement in their symptoms although the eyelid margins were only completely cleared of lashes in three cases. Residual lashes were not treated per se but only if significant discomfort or signs of irritation were present. Eleven patients in this group had follow up of more than 2 years: average 4.5 years (range 2 to 9 years), four patients had follow up less than 1 year owing to recent surgery or were unable to be reviewed. Since only two patients required retreatment of lashes (by electrolysis and excision of lash roots respectively), we report an $87 \%$ success rate for lid splitting with posterior lamellar cryotherapy for congenital distichiasis.

Following lid split with cryotherapy, eyelid oedema settled in most cases within 4 to 8 weeks. One patient remained uncomfortable for 6 months. One patient developed pseudoepithelial hyperplasia of the eyelid margin which is thought to have occurred because the two lid lamellae were not sutured but left to granulate following lid splitting. Although uncosmetic, the patient was comfortable, no further treatment was required and the lid margin eventually returned to normal. Following this case, the anterior lamella has always been sutured to the advanced posterior lamella with an absorbable suture and this problem has not recurred. Overadvancement of the posterior lamella occurred in one patient. This cosmetic problem was corrected by shaving down the advanced posterior lamella to restore a normal lid margin appearance. On long term review ( 3 to 11 years), keratin was found to develop along the posterior lid margin in three patients. A course of retinoic acid $0.01 \%$ was used successfully to remove the keratin in one patient without any adverse reactions. Two patients will undergo similar retinoic acid treatment. In cases that underwent lid splitting and cryotherapy, the long term postoperative appearance of the tarsus at the lid margin was found to be approximaely half to two thirds normal thickness.

\section{Discussion}

There have been a considerable number of different treatments tried for permanent cure of this condition. Electrolysis and cryotherapy remain the simplest forms of treatment for distichiasis with only minimal lid margin involvement. For more extensive distichiasis, cryotherapy with nitrous oxide applied to the conjunctival side of the lid margin effectively removes the unwanted posterior lashes ${ }^{4}$ but was found to depigment the normal anterior lashes. ${ }^{5}$

Surgical excision of the lash roots has been performed individually, ${ }^{6}$ via a trapdoor in the conjunctival surface of the tarsal plate, ${ }^{7}$ and via resection of the distal part of the tarsal plate. ${ }^{8-11}$
Individual lash root excision is performed via a razor incision over the distichiatic lash root. However, it is time consuming and risks damaging the posterior lid margin. Excision of distichiasis lash roots through a trapdoor incision in the tarsoconjunctival surface just posterior to the eyelid margin has been reported to cause less trichiasis, entropion, and eyelid margin distortion but nevertheless scars the important posterior conjunctival surface of the eyelid. Resection of the distal tarsal plate has been tried with simple advancement of the cut edge to form a new posterior lid margin. ${ }^{8}$ Replacement of the resected distal posterior lid margin has been tried with nasal chondromucosa, ${ }^{9}$ and buccal mucous membrane. ${ }^{1011}$

Cryotherapy is an excellent means of ablating lash roots. However, more than half of the patients treated with simple cryotherapy/ electrolysis required retreatment of some kind. Eyelids with distichiasis tend to have the lashes evenly distributed along the length of the lid margin necessitating extensive treatment. Cryotherapy is better performed and the complications of lash and skin depigmentation can be avoided with the technique of lid splitting at the grey line with cryotherapy to the posterior lamella. This treatment is effective, does not depigment the skin or lashes of the anterior lamella, prevents loss of normal anterior lashes, and does not disturb the posterior lid margin. Theoretically cryotherapy of the posterior lamella may cause entropion with trichiasis. This is prevented by the anterior lamellar recession and advancement of the posterior lamella. No complications of entropion or lid margin distortion were found to occur in this series. Although eyelid erythema and swelling persisted for several months, all except one case fully resolved with the latter improving at last follow up visit. The technique of lid splitting and cryotherapy is relatively straight forward but familiarity with upper lid retractor anatomy is necessary. We suggest that this is the procedure of choice for distichiasis with extensive lid margin involvement.

Complete removal of lashes occurred after lid split and cryotherapy in only three patients, yet retreatment after this procedure was only required in two patients. In young children in whom history was unreliable or examination difficult, no further treatment was carried out if symptoms or signs were minimal. There was often such a dramatic improvement in the child's postoperative comfort that this may have made any postoperative minor irritation observed by the parents seem insignificant. In older patients, it was found that a few remaining lashes were most often asymptomatic or caused so little in the way of irritation, that the patient declined any further treatment. The incomplete destruction of the lash roots most probably occurs secondary to inadequate temperature or duration of the freeze. Inaccurate placement of the thermocouple or calibration may account for the former.

The associated findings with distichiasis in this series were epicanthus, telecanthus, blepharophimosis, ptosis, colour blindness, lymphoedema, eyelid oedema, and the Pierre Robin syndrome. To the authors' knowledge, 
the associations of idiopathic eyelid oedema and Pierre Robin syndrome have not previously been reported. Distichiasis has been previously reported with hereditary spinal arachnoid cysts, ${ }^{12}$ lower lid ectropion, ${ }^{12}$ reduced serum cholinesterase, ${ }^{13}$ cleft palate, ${ }^{14}$ trisomy, ${ }^{15} 18$ epicanthus, ${ }^{16}$ blepharophimosis, microphthalmos, dystrophic retinal pigmentation, and optic disc pallor in the oculo-cerebro-renal syndrome, ${ }^{17}$ ectrodactyly, ${ }^{18}$ corneal hypoaesthesia, ${ }^{19}$ uvula bifida, and submucous cleft of the palate. ${ }^{20}$ Distichiasis has been reported with both late onset Meige type lymphoedema ${ }^{1221}$ and early onset Milroy type lymphoedema..$^{22}$ The lymphoedema associated with distichiasis has been found to be bilateral hyperplasia by lymphography. ${ }^{23}$ The association of eyelid oedema has not previously been reported.

The inheritance pattern of distichiasis has been previously reported as autosomal dominant. ${ }^{24} 25$ Fifty percent of patients in this study gave a family history of distichiasis. Three patients, with more than one previous generation affected, displayed an autosomal dominant pattern. Thirty percent of patients gave a history of distichiasis and lymphoedema. The average age in this series was less than 10 years and since the onset of lymphoedema tends to occur in adulthood, this would explain why few patients were seen with coexistent lymphoedema in this series. In families with distichiasis and lymphoedema affecting more than one previous generation, the distichiasis tended to occur in association with lymophoedema in family members affected rather than the distichiasis occurring alone.

No royalties are received by the author, J R O Collin, from Keeler Ophthalmic Instruments from the sales of the Collin cryoprobe.

1 Anderson RL, Harvey JT. Lid splitting and posterior lamellar cryosurgery for congenital and acquired distichiasis. Arch Ophthalmol 1981; 99: 631-4.
2 Collin JRO. A manual of systemic eyelid surgery. 2nd Ed. Edinburgh: Churchill Livingstone, 1989; 25-6.

3 Johnson RLC, Collin JRO. Treatment of trichiasis with a lid cryoprobe. Br f Ophthalmol 1985; 69; 267-70.
collin JRO. Treatment of trich

4 Matthaus W, Matthes R. Technik und Langzeitergebnisse der kryoepilation bei tichiasis end distichiasis. Klin Monatsbl Augenheilkd 1990; 196: 59-61.

5 Freuh B. Treatment of distichiasis with cryotherapy. Ophthalmic Surg 1981; 12: 100-3.

6 Wolfley $\mathrm{D}$. Excision of individual follicles for the management of congenital distichiasis and localised trichiasis. $\mathcal{F}$ Paed Ophthalmol Strabismus 1987; 24: 22-6.

7 Dortzbach RK, Butera RT. Excision of distichiasis eyelashes through a tarso-conjunctival trapdoor. Arch Ophthalmol 1978; 96: 111-2.

8 Fox SA. Distichiasis. Am f Ophthalmol 1962; 53: 14-7.

9 Rodallec A, Krastinova D. Son interet dans la correction de l'entropion par cicatrice tarso-conjunctivale, du trichiasis et du distichiasis. La grette chondro-muqueuse. $\mathcal{f}$ Fr Ophtalmol 1983; 6: 87-93.

mol 1983; 6: 87-93.
10 Metaioreau JP. Traitment du distichiasis par la marginoplasty palpebrale. Bull Soc Ophtalmol Fr 1985; 85: 1247-8.

11 White JH. Correction of distichiasis by tarsal resection and mucous membrane grafting. Am $\mathcal{F}$ Ophthalmol 1975; 80: 507-8.

12 Schwartz JF, O'Brien MS, Hoffman JC Jr. Hereditary spinal arachnoid cysts, distichiasis, and lymphoedema. Ann Neuro 1980; 7: 340-3.

13 Shammas HF, Tabbarra KF, Der Kaloustan VM. Atypical serum cholesterase in a family with congenital distichiasis. f Med Genet 1976; 13: 514-5.

14 Bartley GB, Jackson IT. Distichiasis and cleft palate. Plastic Reconstruct Surg 1989; 84: 129-32.

15 Mehta L, Shannon RS, Duckett DP, Young ID. Trisomy 18 in a 13 year old girl. $\mathcal{F}$ Med Genet 1986; 23: 256-78.

16 Deutsche AR. Distichiasis and epicanthus. A study of one family. Tennessee Med Assoc 1969; 62: 1023-5.

17 Brancato $R$, Matteini M, Cotrozzi G. Sindrome Oculocerebro-renale in soggott portatore di blefarophimosi e di distichiasisi. Ann Ottal 1965; 91: 35-57.

18 Collier MM. Ecrodactylie droite et distichiasis inferieur bilateral. Bull Soc D'Ophtalmol 1964; 64: 49-52.

19 Kremer I, Weinberger D, Cohen S, Ben Sira I. Corneal hypoaesthesia in asymptomatic familial distichiasis. BrF Ophthalmol 1986; 70: 132-4.

20 Jester HG. Lymphoedema distichiasis. A rare hereditary syndrome. Hum Genet 1977; 39: 113-6.

21 Campbell KN. Milroy's disease. Chronic hereditary oedema. Univ Bull Ann Arbor 1945; 11: 69-70.

22 Kolin T, Johns KJ, Wadlington WB, Butler MG, Sunlap MA, Wright $\mathrm{KW}$. Hereditary lymphoedema and distichiasis. Arch Ophthalmol 1991; 109: 980-1.

23 Dale RF. Primary lymphoedema when found with distichiasis is of the type defined as bilateral hyperplasia by lymphography. $\mathcal{F}$ Med Genetics 1987; 24: 170-1.

24 Mustonen E. Congenital distichiasis. A report of eleven cases and an investigation of the mode of inheritance. Acta Ophthalmol 1972; 50: 598-607.

25 Holmes LB, Fields JP, Zabriskie JB. Hereditary late-onset lymphoedema. Paediatrics 1978; 61: 575-9. 